\title{
Primer Reporte de la Dieta del Búho Orejicorto Asio flammeus (Strigiformes: Strigidae) en Pichincha, Ecuador
}

\author{
Glenda M. Pozo-Zamora ${ }^{1 *}$, Jorge Brito ${ }^{1,2}$, Rubí García $^{1}$, Ibeth Alarcón ${ }^{3}$, Héctor Cadena-Ortiz ${ }^{1,4}$ \\ ${ }^{1}$ Museo Ecuatoriano de Ciencias Naturales del Instituto Nacional de Biodiversidad, Calle Rumipamba 341 y Av. de los Shyris, \\ Casilla: 17-07-8976, Quito, Ecuador. \\ ${ }^{2}$ Instituto de Ciencias Biológicas, Escuela Politécnica Nacional, Calle Ladrón de Guevara y Veintimilla, Casilla: 17-01- 2759, \\ Quito, Ecuador. \\ ${ }^{3}$ Aves y Conservación/BirdLife Ecuador, Área de Investigación y Monitoreo de Avifauna, Joaquín Tinajero E3-05 y Jorge Drom, \\ Quito, Ecuador. \\ ${ }^{4}$ Aves Quito (Club de Observadores de Aves), Ecuador \\ *Autor para correspondencia/Corresponding author,e-mail: glenda.pozo@yahoo.es \\ Editado por/Edited by: Juan F. Freile. \\ Recibido/Received: 21-07-2016. Aceptado/Accepted: 01-03-2017. \\ Publicado en línea/Published on Web: 18-04-2017.
}

DOI:

First report of the diet of Short-eared Owl Asio flammeus (Strigiformes: Strigidae) in Pichincha, Ecuador

\section{Resumen}

Presentamos información sobre la dieta del Búho Orejicorto Asio flammeus en la provincia de Pichincha, Ecuador. Analizamos 52 egagrópilas, colectadas entre 2014 y 2016 en tres localidades rurales. Encontramos 167 ítems presas de 22 especies. La dieta incluyó mamíferos pequeños (50,8\%), seguida de insectos $(43,1 \%)$, aves $(4,7 \%)$ y anfibios y reptiles $(0,5 \%$ cada uno). El mayor aporte de biomasa fue proporcionado por los mamíferos (87\%). La dieta de A. flammeus en Pichincha es similar a aquellas reportadas para la especie en otras áreas a lo largo de América.

Palabras Clave. Alimentación, egagrópilas, historia natural, presas.

\begin{abstract}
We present information on the diet of Short-eared Owl Asio flammeus from the province of Pichincha, Ecuador. We analyzed 52 pellets, collected between 2014 and 2016 in three rural localities. We found 167 prey items of 22 prey species. The diet included small mammals $(50.8 \%)$, insects $(43.1 \%)$, birds $(4.7 \%)$, and amphibians and reptiles $(0.5 \%$ each). The greatest biomass was contributed by mammals ( $87 \%$ ). The $\operatorname{diet}$ of A. flammeus in Pichincha is similar to those reported for the species in other areas throughout America.
\end{abstract}

Keywords. Diet, natural history, pellets, preys. 


\section{INTRODUCCIÓN}

El conocimiento de la ecología trófica de las especies es esencial para comprender las estrategias de alimentación, dinámicas de nicho, interacciones intra e interespecíficas y división trófica (Marti et al., 1993; Bellocq, 2000). El Búho Orejicorto Asio flammeus es una especie con amplia distribución mundial (König \& Weick, 2008). Su dieta ha sido estudiada en varios continentes, incluyendo Europa (Roberts \& Bowman, 1986; Delibes et al., 1991; Cullen \& Smiddy, 2012; Kleefstra et al., 2015), África (Djilali et al., 2016), Asia (Lin \& Yeh, 2002; Jathar et al., 2011) y Norteamérica (Banfield, 1947; Clark 1975; Holt, 1993; Hogan et al., 1996; Reid et al., 2011; Williford et al., 2011). En América del Sur, los hábitos tróficos de A. flammeus han sido estudiados en Colombia (Borrero 1962), Chile (Rau et al., 1992; Fuentes et al., 1993; Martínez et al., 1998), Argentina (Diéguez, 1996; Cirignoli et al., 2001; Bó et al., 2007; Baladrón et al., 2014) y Paraguay (Torres et al., 2014).

El estado de conocimiento de las aves rapaces nocturnas en Ecuador es limitado (Freile et al., 2012). Existen escasos estudios sobre la ecología trófica (Moreno, 2010; Cadena-Ortiz et al., 2013; Moreno \& Román, 2013; Brito et al., 2015; Cadena-Ortiz et al., 2016) y ninguno a largo plazo (Freile et al., 2015). La única información disponible sobre la dieta de Asio flammeus en Ecuador proviene de la subespecie Asio flammeus galapagoensis, endémica de las islas Galápagos (de Groot, 1983). En esta publicación damos a conocer por primera vez información sobre la dieta de Asio flammeus en Ecuador continental.

\section{MÉTODOS}

Entre septiembre 2014 y junio 2016, colectamos egagrópilas de Asio flammeus durante visitas esporádicas a tres localidades de moderada alteración antrópica en la provincia de Pichincha (todas ubicadas en el piso zoogeográfico Templado; Albuja et al., 2012): (1) Yanacocha $\left(0,103^{\circ} \mathrm{S} ; 78,590^{\circ} \mathrm{O}, 3398 \mathrm{~m}\right)$, en zonas de pastizales abandonados; (2) áreas adyacentes al Aeropuerto Internacional Mariscal Sucre (AIMS) en Tababela $\left(0,119^{\circ} \mathrm{S} ; 78,361^{\circ} \mathrm{O}, 2371 \mathrm{~m}\right)$, en arbustales con vegetación nativa de bosque seco; y (3) hacienda Tambillo Alto $\left(0,410^{\circ} \mathrm{S} ; 78,555^{\circ} \mathrm{O}, 2834 \mathrm{~m}\right)$, en pastizales que bordean remanentes de bosque montano. En los tres sitios buscamos egagrópilas de manera activa en perchas y dormideros (aunque sin encontrar sitios de nidificación). En estas visitas observamos individuos adultos de A. flammeus forrajeando, perchando o volando en los alrededores de los sitios de colección de egagrópilas (Fig. 1). En total recolectamos 52 egagrópilas: 25 en Yanacocha, 21 en AIMS, y 6 en Tambillo. Secamos las egagrópilas a temperatura ambiente y tomamos medidas de longitud y ancho (en $\mathrm{mm}$ ) utilizando un calibrador digital Buffalo Tools, con precisión de $\pm 0,01 \mathrm{~mm}$. Pesamos cada egagrópila en una balanza analítica Fisher Scientific $120 \mathrm{~g} \times 0,001 \mathrm{~g}$. Todos los valores son expresados como media \pm desviación estándar (rango). El contenido de las egragópilas fue separado, etiquetado por localidad con códigos únicos, y depositado en la colección del Museo Ecuatoriano de Ciencias Naturales (MECN), Quito.

Utilizamos las siguientes referencias para identificar los restos de mamíferos y para obtener la masa promedio de los taxones: Hershkovitz (1962), Carleton \& Musser (1989), Tirira (2007), Weskler \& Percequillo (2011), Moreno \& Albuja (2014). Para obtener la masa de los reptiles utilizamos la información de Ramírez-Jaramillo et al. (2015). En el caso de los mamíferos, aves e insectos también comparamos con especímenes del MECN.

Debido al pequeño tamaño de muestra por localidad, todas las egagrópilas fueron analizadas de manera conjunta. Para cada ítem presa consumido calculamos el número mínimo de individuos (NMI), contando únicamente el número de pares de mandíbulas en insectos o restos de cráneos en aves y mamíferos con el fin de evitar reconteo (Manning \& Jones, 1990). Obtuvimos la biomasa de cada ítem presa multiplicando la masa media (en g) de cada especie por el NMI correspondiente (Herrera \& Jaksic, 1980). Realizamos una correlación lineal simple entre el tamaño de la presa y su frecuencia en las egagrópilas mediante el programa Past 3.1 (Hammer et al., 2011).

\section{RESULTADOS}

Las egagrópilas midieron 44,0 \pm 5,9 $\mathrm{mm}(26,1-60,5 \mathrm{~mm})$ de longitud, 23,3 \pm 4,8 $\mathrm{mm}(16,1-33,1 \mathrm{~mm})$ de ancho y 2,3 $\pm 0,7 \mathrm{~g}(1,0-6,02 \mathrm{~g})$ de masa. El número promedio de presas por egagrópila fue 2,6 $\pm 1,1(1-5)$. En total encontramos 167 individuos presas de 22 especies. Los mamíferos fueron las presas más frecuentes en la dieta de Asio flammeus (50,8 \% de todas los individuos), seguido por insectos $(43,1 \%)$, aves $(4,7 \%)$ y anfibios y reptiles $(0,5 \%$, cada uno). Entre los mamíferos, los roedores fueron el grupo más consumido (75,2\% del total de mamíferos), siendo las especies más frecuentes Phyllotis haggardi, Akodon sp. (cf. mollis) y Reithrodontomys soederstroemi (Tabla 1). Los mamíferos hicieron la mayor contribución energética a la dieta de A. flammeus, con casi $87 \%$ de la biomasa total (Tabla 1). La 
contribución de biomasa de las aves alcanzó un poco más del $10 \%$, mientras que aquella de anfibios, reptiles e insectos fue mínima (Tabla 1). No encontramos relación entre la masa de las presas y su frecuencia en las egagrópilas $\left(\mathrm{r}^{2}=\right.$ $-0,042, \mathrm{p}=0,359)$.

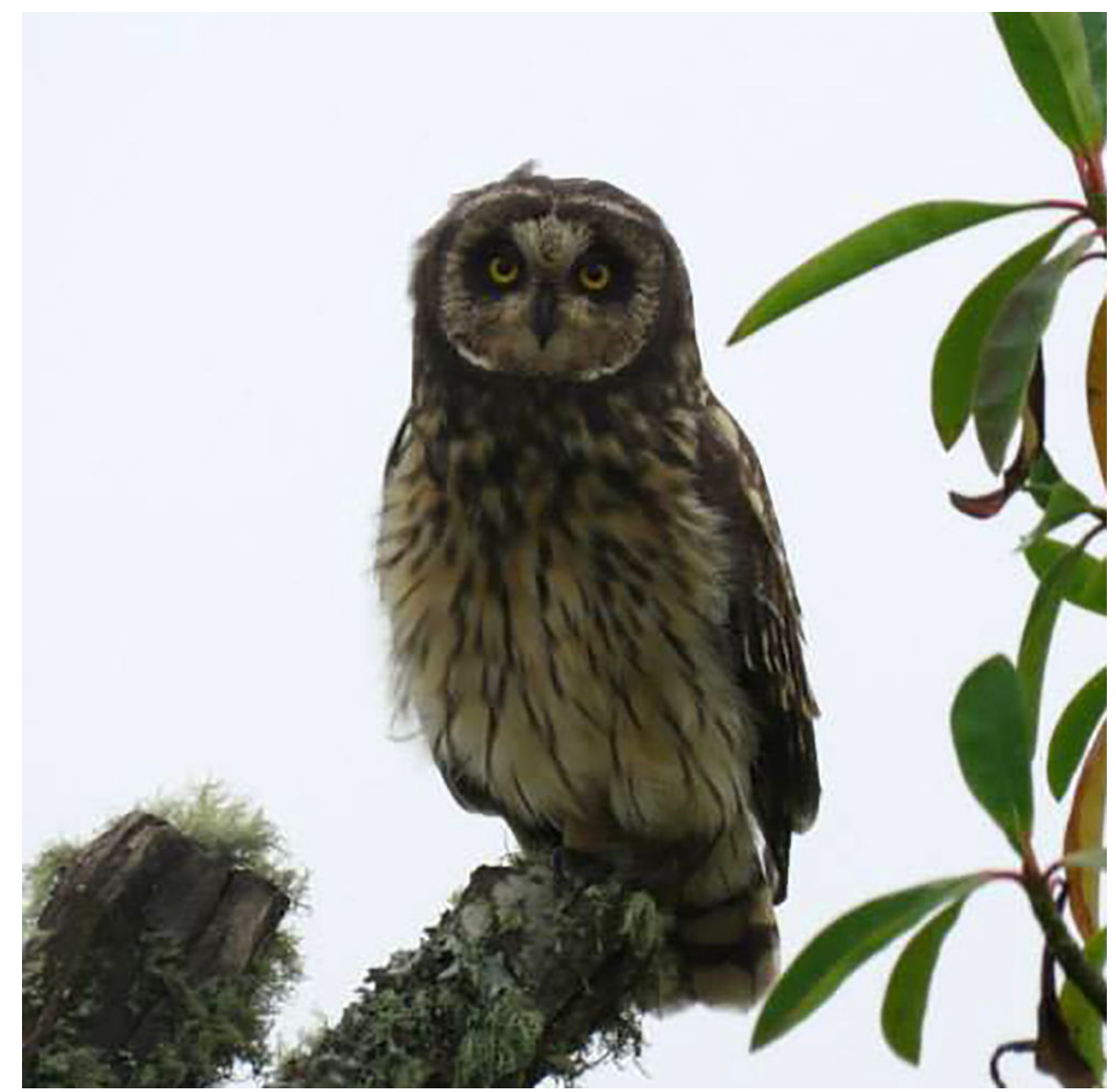

Figura 1: Búho Orejicorto Asio flammeus perchado en la localidad de Yanacocha, provincia de Pichincha, Ecuador. Fotografía: T. Santander.

Tabla 1: Presas del Búho Orejicorto Asio flammeus en tres localidades en la provincia de Pichincha, Ecuador. Localidades: Yanacocha (Y), Aeropuerto Internacional Mariscal Sucre (AIMS) y Tambillo Alto (TA).

\begin{tabular}{|c|c|c|c|c|c|c|}
\hline \multirow{2}{*}{ Orden/Especie } & \multicolumn{3}{|c|}{ Localidades } & \multirow{2}{*}{$\begin{array}{l}\text { Masa } \\
(\text { en g) }\end{array}$} & \multirow{2}{*}{$\begin{array}{c}\text { Individuos } \\
(\%)\end{array}$} & \multirow{2}{*}{$\begin{array}{l}\text { Biomasa } \\
\text { en g( \%) }\end{array}$} \\
\hline & $\mathbf{Y}$ & AIMS & TA & & & \\
\hline MAMMALIA & & & & & $85(50,8)$ & $\begin{array}{c}2016,6 \\
(86,5)\end{array}$ \\
\hline $\begin{array}{l}\text { PAUCITUBERCULATA } \\
\text { Caenolestes fuliginosus } \\
\text { RODENTIA }\end{array}$ & 1 & - & - & 28 & $1(0,6)$ & $28(1,2)$ \\
\hline Reithrodontomys soederstroemi & 1 & 12 & - & 15 & $13(7,8)$ & $195(8,4)$ \\
\hline Akodon sp. (cf. mollis) & 8 & 5 & 1 & 15 & $14(8,4)$ & $210(9,0)$ \\
\hline Phyllotis haggardi & 7 & 8 & - & 20 & $15(9,0)$ & $300(12,9)$ \\
\hline Thomasomys baeops & 1 & - & - & 36 & $1(0,6)$ & $36(1,5)$ \\
\hline Microryzomys altissimus & 6 & - & - & 16 & $6(3,6)$ & $96(4,1)$ \\
\hline Thomasomys paramorum & 2 & - & - & 25 & $2(1,2)$ & $50(2,1)$ \\
\hline Mus musculus & - & 2 & 2 & 14 & $4(2,4)$ & $56(2,4)$ \\
\hline
\end{tabular}




\begin{tabular}{|c|c|c|c|c|c|c|}
\hline \multirow{2}{*}{ Orden/Especie } & \multicolumn{3}{|c|}{ Localidades } & \multirow{2}{*}{$\begin{array}{l}\text { Masa } \\
\text { (en g) }\end{array}$} & \multirow{2}{*}{$\begin{array}{c}\text { Individuos } \\
(\%)\end{array}$} & \multirow{2}{*}{$\begin{array}{l}\text { Biomasa } \\
\text { en } g(\%)\end{array}$} \\
\hline & $\mathbf{Y}$ & AIMS & $\mathbf{T A}$ & & & \\
\hline \multicolumn{7}{|l|}{ CHIROPTERA } \\
\hline Anoura sp. (cf. fistulata) & 2 & - & - & 12,7 & $2(1,2)$ & $25,4(1,1)$ \\
\hline Anoura peruana & 4 & - & - & 15,3 & $4(2,4)$ & $61,2(2,6)$ \\
\hline $\begin{array}{l}\text { Dermanura sp. } \\
\text { LAGOMORPHA }\end{array}$ & 3 & - & - & 13 & $3(1,8)$ & $39(1,7)$ \\
\hline $\begin{array}{l}\text { Sylvilagus andinus (crías) } \\
\text { EULIPOTYPHLA }\end{array}$ & 1 & 11 & - & 70 & $12(7,2)$ & $840(36,0)$ \\
\hline Cryptotis equatoris & 2 & - & - & 10 & $2(1,2)$ & $20(0,9)$ \\
\hline Cryptotis osgoodi & 3 & - & 3 & 10 & $6(3,6)$ & $60(2,6)$ \\
\hline AVES & & & & & $8(4,7)$ & $261(11,3)$ \\
\hline PASSERIFORMES & 2 & & & & & \\
\hline Zonotrichia capensis & - & 4 & - & 22 & $4(2,4)$ & $88(3,8)$ \\
\hline Phrygilus plebejus & - & 2 & - & 15 & $2(1,2)$ & $30(1,3)$ \\
\hline Turdus fuscater & 1 & - & - & 130 & $1(0,6)$ & $130(5,6)$ \\
\hline Catamenia sp. & 1 & & & 13 & $1(0,6)$ & $13(0,6)$ \\
\hline ANURA & & & & & $1(0,5)$ & $0,9(0,1)$ \\
\hline Pristimantis unistrigatus & 1 & - & - & 0.9 & $1(0,6)$ & $0,9(0,1)$ \\
\hline $\begin{array}{l}\text { REPTILIA } \\
\text { SAURIA }\end{array}$ & & & & & $1(0,5)$ & $11(0,1)$ \\
\hline $\begin{array}{l}\text { Sternocercus guentheri } \\
\text { INSECTA }\end{array}$ & - & 1 & - & 11 & $\begin{array}{c}1(0,6) \\
\mathbf{7 2}(\mathbf{4 3 , 1 )}\end{array}$ & $\begin{array}{c}11(0,1) \\
45(\mathbf{1 , 2 6})\end{array}$ \\
\hline COLEOPTERA & & & & & & \\
\hline Barotheus andinus & 48 & 2 & 4 & 0,5 & $54(32,3)$ & $27(1,16)$ \\
\hline Heterogomphus bourcieri & 18 & - & - & 1 & $18(10,8)$ & $18(0,1)$ \\
\hline Total individuos & 94 & 47 & 10 & & $167(100)$ & $2334,5(100)$ \\
\hline
\end{tabular}

\section{DISCUSIÓN}

Nuestro estudio indica que la dieta de Asio flammeus en Pichincha, Ecuador, está compuesta principalmente por roedores, mayormente especies nativas. Estos resultados coinciden con lo reportado por otros estudios llevados a cabo a lo largo del continente americano (Banfield, 1947; Clark 1975, Rau et al., 1992; Hogan et al., 1996; Martínez et al., 1998; Cirignoli et al., 2001; Reid et al., 2011; Williford et al., 2011; Baladrón et al., 2014; Torres et al., 2014). No obstante, se ha reportado que las ratas del género Rattus pueden hacer una importante contribución de biomasa en la época invernal en áreas continentales (Martínez et al., 1998), mientras que en áreas insulares parece consumir mayormente roedores introducidos y aves (de Groot, 1983; Fuentes et al., 1993; Cullen \& Smiddy, 2012).

König \& Weick (2008) sugirieron que Asio flammeus se alimenta de murciélagos de manera poco frecuente. Sin embargo, Torres et al. (2014) y en este estudio evidenciamos el consumo de murciélagos. Al parecer, A. flammeus puede ser un depredador importante de quirópteros si estos son abundantes (Djilali et al., 2016).

Varias de las especies de mamíferos y reptiles consumidos por Asio flammeus en nuestra área de estudio (e.g. Reithrodontomys soderstromi, Phyllotis haggardi, Sylvilagus andinus, Sternocercus guentheri) también son presas de otros depredadores simpátricos como Lycalopex culpaeus (Trujillo \& Trujillo, 2007; Reina, 2013), Tyto alba (Moreno \& Román, 2013) y Athene cunicularia (Cadena-Ortiz et al., 2016). El solapamiento del nicho trófico sugiere que estas presas tienen poblaciones (localmente) numerosas, que son especies particularmente susceptibles de ser cazadas o que hay preferencia de los depredadores hacia estas presas.

Para definir el comportamiento de forrajeo de Asio flammeus es necesario estudiar simultáneamente la abundancia y tamaño de presas mediante la recolección de egagrópilas (Jaksic, 1989). Asio flammeus ha sido considerado como especialista en mamíferos y aves por su preferencia tanto numérica como de biomasa por estos grupos (Figueroa et al., 2015). Sin embargo, nuestra muestra sugiere una especialización en mamíferos. La falta de correlación entre el tamaño de las presas y su frecuencia en las egagrópilas sugiere que el tamaño de presas manipulable por A. flammeus corresponde a mamíferos pequeños, menores a $70 \mathrm{~g}$. 


\section{AGRADECIMIENTOS}

Agradecemos a Quiport por financiar el monitoreo biológico en áreas de influencia del AIMS y a César Garzón por coordinar la logística durante la fase inicial del estudio; a Jenny Curay y Rocío Vargas por su asistencia en el trabajo de laboratorio en el MECN; a Tatiana Santander y Esteban Guevara, de Aves y Conservación, por su apoyo en Yanacocha dentro del proyecto "Hacia la conservación participativa de los bosques alto andinos del nor-occidente de Ecuador"; a la familia Páez, propietarios de la finca en Yanacocha, y a Wilson Hipo por su ayuda y observaciones en campo; y a Ricardo Figueroa y un revisor anónimo por sus acertados comentarios para el mejoramiento del manuscrito. El Ministerio del Ambiente facilitó el permiso de investigación No. 01-2014-RIC-FAU-FLO-DPAP-MA, para el trabajo en el AIMS.

\section{REFERENCIAS}

Albuja, L., Almendáriz, A., Barriga, R., Montalvo, L. D., Cáceres, F., \& Román, J. L. (2012). Fauna de Vertebrados del Ecuador. Quito: Instituto de Ciencias Biológicas, Escuela Politécnica Nacional.

Baladrón, A. V., Cavalli, M., \& Cardoni, D. A. (2014). Presas del nuco (Asio flammeus) en un área de pastizal pampeano de Argentina. Boletín Chileno de Ornitología, 20(1-2), 33-36. Recuperado de: http://aveschile.cl/web/wp-content/uploads/2015/07/ Baladr\%C3\%B3n-et-al..pdf

Banfield, A. W. F. (1947). A study of the winter feeding habits of the Short-eared Owl (Asio flammeus) in the Toronto Region. Canadian Journal of Research, 25(2), 45-65. doi: http://doi.org/10.1139/cjr47d-003

Bellocq, M. I. (2000). A review of the trophic ecology of the Barn Owl in Argentina. Journal of Raptor Research, 34(2), 108119. Recuperado de: https://sora.unm.edu/node/53810

Bó, M. S., Baladrón, A. V., \& Biondi, L. M. (2007). Ecología trófica de Falconiformes y Strigiformes: tiempo de síntesis. El Hornero, 22(2), 97-115. Recuperado de: http://ref.scielo.org/qp5cdf

Borrero, J. I. (1962). Notas varias sobre Asio flammeus bogotensis en Colombia. Revista de Biología Tropical, 10(1), 45-59. Recuperado de: http://www.ots.ac.cr/rbt/attachments/volumes/vol10-1/06-Borrero-Asio.pdf

Brito, J., Orellana-Vásquez, H., Cadena-Ortiz, H., Vargas, R., Pozo-Zamora, G., \& Curay, J. (2015). Mamíferos pequeños en la dieta de la lechuza Tyto alba (Strigiformes: Tytonidae) en dos localidades del occidente de Ecuador, con ampliación distribucional de Ichthyomys hydrobates (Rodentia: Cricetidae). Papéis Avulsos de Zoologia, 55(19), 261-268. doi: http://dx.doi.org/10.1590/ 0031-1049.2015.55.19

Cadena-Ortiz, H., Freile, J. F., \& Bahamonde-Vinueza, D. (2013). Información sobre la dieta de algunos búhos (Strigidae) del Ecuador. Ornitología Neotropical, 24(4), 469-474. Recuperado de: https://sora.unm.edu/node/133393

Cadena-Ortiz, H., Garzón, C., Villamarín-Cortéz, S., Pozo-Zamora, G. M., Echeverría-Vaca, G., Yánez, J., \& Brito-M, J. (2016). Diet of the Burrowing Owl Athene cunicularia, in two locations of the inter-Andean valley Ecuador. Revista Brasileira de Ornitología, 24(2), 122-128. Recuperado de: http://www4.museu-goeldi.br/revistabrornito/revista/index.php/BJO/article/view/1153/pdf_ 960

Carleton, M. D., \& Musser, G. G. (1989). Systematic studies of Oryzomyine rodents (Muridae, Sigmodontinae): a synopsis of Microryzomys. Bulletin of the American Museum of Natural History, 191, 1-83. Recuperado de: http://digitallibrary.amnh.org/handle/2246/953?show=full

Cirignoli, S., Podestá, D. H., \& Pardiñas, U. F. J. (2001). Diet of the Short-eared Owl in northwestern Argentina. Journal of Raptor Research, 35(1), 68-69. Recuperado de: https://sora.unm.edu/sites/default/files/journals/jrr/v035n01/p00068-p00069.pdf

Clark, R. J. (1975). A field study of the Short-eared Owl, Asio flammeus (Pontoppidan), in North America. Wildlife Monographs, 47, 3-67. Recuperado de: http://www.jstor.org/stable/3830422

Cullen, C., \& Smiddy, P. (2012). Diet of Short-eared Owls Asio flammeus over seven winters in country Cork, Ireland. Biology and Environment: Proceedings of the Royal Irish Academy, 112B(2), 217-223. Recuperado de: http://www.jstor.org/stable/41714033

De Groot, R. S. (1983). Origin, status and ecology of the owls in Galapagos. Ardea, 71, 167-182. Recuperado de: http://ardea. nou.nu/ardea_show_abstract.php?lang=nl\&nr=957

Delibes, J., Hiraldo, F., \& Heredia, B. (1991). Datos sobre la dieta invernal de la Lechuza Campestre (Asio flammeus) en un periodo de abundancia de Topillo Campesino (Microtus arvalis) en la Submeseta Norte (España). Ecología, 5: 355-358. Recuperado de: http://www.mapama.gob.es/gl/parques-nacionales-oapn/publicaciones/ecologia_05_28_tcm10-45780.pdf 
Diéguez, A. J. (1996). Aves depredadas por Asio flammeus suinda - 1 - en Saladillo, Provincia de Buenos Aires. APRONA Boletín Científico Asociación para la Protección de la Naturaleza, IX (30), 25-26. Recuperado de: http://www.sarem.org.ar/wp-content/ uploads/2015/08/BoletinAPRONA_30_ago1996.pdf

Djilali, K., Sekour, M., Souttou, K., Ababsa, L., Guezoul, O., Denys, C., \& Doumandji, S. (2016). Diet of Short-eared Owl Asio flammeus (Pontoppidan, 1763) in desert area at Hassi El Gara (El Golea, Algeria). Zoology and Ecology, 26(6), 159-165. doi: http:// dx.doi.org/10.1080/21658005.2016.1184907

Figueroa, R. A., Rau, J. R., Mayorga, S., Martínez, D. R., Corales, E. S., Mansilla, A., \& Figueroa, R. (2009). Rodent prey of the barn owl Tyto alba and short-eared owl Asio flammeus during winter in agricultural lands in southern Chile. Wildlife Biology, 15(2), 129-136. doi: http://doi.org/10.2981/08-005

Figueroa, R. A., Alvarado, S., Corales, E. S., González-Acuña, D., Schlatter, R., \& Martínez, D. R. (2015). Los Búhos de Chile. En: P. L. Enríquez (Ed), Los Búhos Neotropicales: diversidad y conservación (pp.173-272). México: Ecosur.

Freile, J. F., Castro, D. F., \& Varela, S. (2012). Estado del conocimiento, distribución y conservación de aves rapaces nocturnas en Ecuador. Ornitología Neotropical, 23(supplement), 235-244. Recuperado de: https://sora.unm.edu/sites/default/files/Freile. pdf

Freile, J. F., Guevara, E., Pacheco, C., \& Santander, T. (2015). Los Búhos del Ecuador. En P. L. Enríquez (Ed), Los Búhos Neotropicales: diversidad y conservación (pp. 333-353). México: Ecosur.

Fuentes, M. A., Simonetti, J. A., Sepúlveda, M. S., \& Acevedo, P. A. (1993). Diet of the Red-backed Buzzard (Buteo polyosoma exsul) and the Short-eared Owl (Asio flammeus suinda) in the Juan Fernández Archipelago of Chile. Journal of Raptor Research, 27(3), 167-169. Recuperado de: https://sora.unm.edu/node/53368

Hammer, ф., Harper, D. A. T., \& Ryan, P. D. (2001). PAST: Paleontological statistics software package for education and data analysis. Palaeontologia Electronica, 4(1), 1-9. Recuperado de: http://palaeo-electronica.org/2001_1/past/issue1_01.htm

Herrera, C. M., \& Jaksic, F. M. (1980). Feeding ecology of the barn owl in Central Chile and southern Spain: a comparative study. Auk, 97(4), 760-767. Recuperado de: http://www.jstor.org/stable/4085747

Hershkovitz, P. (1962). Evolution of Neotropical cricetine rodents (Muridae) with special reference to the Phyllotine group. Fieldiana Zoology, 46, 1-524. doi: http://dx.doi.org/10.5962/bhl.title.2781

Hogan, K. M., Hogan, M. L., Gable, J., \& Bray, M. (1996). Notes on the diet of Short-eared Owls (Asio flammeus) in Texas. Journal of Raptor Research 30(2), 102-104. Recuperado de: https://sora.unm.edu/node/53537

Holt, D. W. (1993). Trophic niche of Nearctic Short-eared Owls. Wilson Bulletin, 105(3), 497-503. Recuperado de: http://www. jstor.org/stable/4163325

Jaksic, S. M. (1989). What do carnivorous predators cue in on: size or abundance of mammalian prey? A crucial test in California, Chile, and Spain. Revista Chilena de Historia Natural, 62(2), 237-249. Recuperado de: http://rchn.biologiachile.cl/pdfs/ 1989/2/Jaksic_1989.pdf

Jathar, G. A., Deshmukh, A. J., Khawale, V. S., Patil, P. S., Pradhan, M. S., Talmale, S. S., Nandvikar, P. D., \& Sawant, A. D. (2011). Winter diet of Short-eared Owls Asio flammeus in Akola, Maharashtra. Indian Birds, 7(1), 17-19. Recuperado de: http:// www.indianbirds.in/pdfs/Short-eared\%20Owls.pdf

Kleefstra, R., Barkema, L., Venema, D. J., \& Spijkstra-Scholten, W. (2015). A plague of voles, an invasion of breeding Shorteared Owls Asio flammeus in the province of Friesland in 2014. Limosa, 88(2), 74-82. Recuperado de: http://www.nou.nu/limosa/ limosa_samenvatting.php?language=UK\&nr=5128

König, C., \& Weick, F. (2008). Owls of the world, second edition. London: Christopher Helm.

Lin, W. L., \& Yeh, C. C. (2002). Winter diet of the Short-eared Owl Asio flammeus (Pontoppidan) at the Augu Farm and the Tatu Rivermouth of Taiwan. Endemic Species Research, 4(2), 63-71.

Manning, R. W., \& Jones, J. K. (1990). Remains of small mammals recovered from Barn Owl pellets from Crosby county, Texas. Texas Journal of Science, 42, 311-312. Recuperado de: http://www.freepatentsonline.com/article/Texas-Journal-Science/128674097. html

Marti, C. D., Steenhof, K., Kochert, M. N., \& Marks, J. S. (1993). Community trophic structure: the doles of diet, body size, 
and activity time in vertebrate predators. Oikos, 67(1), 6-18. doi: http://doi.org/10.2307/3545090

Martínez, D. R., Figueroa, R. A., Ocampo, C. L., \& Jaksic, F. M. (1998). Food habits and hunting ranges of Short-eared Owls (Asio flammeus) in agricultural landscapes of southern Chile. Journal of Raptor Research, 32(2), 111-115. Recuperado de: https:// sora.unm.edu/node/53667

Moreno, P. (2010). Mamíferos presentes en la dieta de la Lechuza de Campanario (Tyto alba) en Valdivia, provincia de Guayas, Ecuador. Avances en Ciencias e Ingenierias, 2(3), B87-B90. doi: http://dx.doi.org/10.18272/aci.v2i3.50

Moreno, P., \& Román, J. L. (2013). Clasificación del género Reithrodontomys en el Ecuador y comentarios sobre la alimentación de la lechuza de campanario (Tyto alba) en los alrededores de Quito. Boletín Técnico, Serie Zoológica, 11(8-9), 16-23. Recuperado de: http://bibdigital.epn.edu.ec/handle/15000/6456

Moreno C., P., \& Albuja, L. (2014). Una nueva especie de musaraña del género Cryptotis Pomel 1848 (Mammalia: Soricomorpha: Soricidae) de Ecuador y estatus taxonómico de Cryptotis equatoris Thomas (1912). Papéis Avulsos de Zoologia, 54(28), 403-418. doi: http://dx.doi.org/10.1590/0031-1049.2014.54.28

Ramírez-Jaramillo, S., Bejarano-Muñoz, P., Rodríguez-Badillo, M., \& Yánez-Muñoz, M. (2015). Uso de perchas nocturnas por Sternocercus guentheri (Iguanidae: Tropidurinae) en dos ecosistemas del distrito metropolitano de Quito (Ecuador). Boletín de la Asociación Herpetológica Española, 26(1), 29-32. Recuperado de: http://www.herpetologica.org/BAHE/BAHE26(1)_\%5B240 \%5D_04_HNat11.pdf

Rau, J. R., Villagra, M. C., Mora, M. L., Martínez, D. R., \& Tillería, M. S. (1992). Food habits of the Short-eared Owl (Asio flammeus) in southern South America. Journal of Raptor Research, 26(1), 35-36. Recuperado de: https://sora.unm.edu/node/53259

Reid, D. G., Doyle, F. I., Kenney, A. L., \& Krebs, C. J. (2011). Some observations of Short-eared Owl, Asio fammeus, ecology on Arctic Tundra, Yukon, Canada. Canadian Field-Naturalist 125(4), 307-315. Recuperado de: http://canadianfieldnaturalist.ca/ index.php/cfn/article/view/1259/1242

Reina, D. S. (2013). Análisis de los componentes alimentarios en la dieta del lobo de páramo Lycalopex culpaeus (Carnivora: Canidae) en el sector de la plataforma del Nuevo Aeropuerto "Mariscal Sucre", parroquia Tababela, cantón Quito, provincia de Pichincha (Tesis de Licenciatura). Escuela de Biología, Universidad Central del Ecuador, Quito, Ecuador.

Roberts, J. L., \& Bowman, N. (1986). Diet and ecology of Short-eared Owls Asio flammeus breeding on heather moor. Bird Study, 33(1), 12-17. doi: http://dx.doi.org/10.1080/00063658609476885

Tirira, D. (2007). Guía de campo de los mamíferos del Ecuador. Quito, Ecuador: Ediciones Murciélago Blanco.

Torres, J. C., Teta, P., \& de la Sancha, N. U. (2014). Presas del Búho Campestre (Asio flammeus) en un Agroecosistema Subtropical de Paraguay. Nuestras Aves, 59, 24-27. Recuperado de: http://hdl.handle.net/11336/9738

Trujillo, F., \& Trujillo, J. (2007). Alimentación del lobo (Lycalopex culpaeus), en el Bosque Protector Jerusalen, GuayllabambaEcuador. Politécnica, Biología, 27(7), 68-75. Recuperado de: http://bibdigital.epn.edu.ec/handle/15000/3838

Weskler, M., \& Percequillo, A. (2011). Key to the genera of the tribe Oryzomyini (Rodentia: Cricetidae: Sigmodontinae). Mastozoología Neotropical, 18(2), 281-292. Recuperado de: http://www.redalyc.org/articulo.oa?id=45722044010

Williford, D., Woodin, M. C., \& Skoruppa, M. K. (2011). The winter diet of Short-eared Owls in subtropical Texas: do southern diets provide evidence of opportunism? Journal of Raptor Research, 45(1), 63-70. doi: http://dx.doi.org/10.3356/JRR-10-40. 1 